\title{
Anti-inflammatory Activity of Chlorella vulgaris in Experimental models of Rats
}

\author{
Swapnil Prakash Chaudhari*, Dheeraj Tukaram Baviskar \\ Institute of Pharmaceutical Education, Boradi, Dhule, Maharashtra, INDIA.
}

\begin{abstract}
Background: The aim of present study is to evaluate the anti-inflmmatory activity of Chlorella vulgaris in acute and chronic experimental inflammatory model. Plant extract was analyzed for photochemical screening of different phytoconstituents. Methods: Paw edema was induced with $1.5 \%$ carrageenan to all the groups except normal. Diclofenac was used as a standard drug and Chlorella vulgaris at 50, 100, $200 \mathrm{mg} / \mathrm{kg}$ dose was used for study. Carrageenan was administered in the paw. Paw thickness was measured at $0,1,2$ and $4 \mathrm{hr}$. Inflammatory cytokines and oxidative stress were measured in paw homogenate. Chronic inflammatory activity was screened by using cotton pellet induced granuloma. Results: In result, paw thickness was increased from $1 \mathrm{hr}$ to $4 \mathrm{hr}$ and Chlorella vulgaris treated group shows decrease in paw thickness. SOD and GSH content was decreased and MDA level increased in carrageenan injected group, however treatment groups shows reverse effects also we found that increase in cytokine level in carrageenan group and decrease in treatment group. In, egg albumin
\end{abstract}

model paw volume was decreased in rats treated with Chlorella vulgaris. In chronic model CV treated group also showed dose dependent activity. Conclusion: From this study we conclude that Chlorella vulgaris has antiinflammatory activity.

Key words: Chlorella vulgaris, Cytokines, Oxidative stress, Inflammation, Paw thickness.

\section{Correspondence}

Mr. Swapnil P Chaudhari,

Head of Department, Institute of Pharmaceutical education, Boradi, TQ- Shirpur, Dhule-425405, Maharashtra, INDIA.

Email id: swapnil4u_2007@rediffmail.com;

ORCID: 0000-0002-6453-4744

DOI: $10.5530 /$ ijpi.2021.4.64

\section{INTRODUCTION}

Inflammation reflects a local reaction to injury from living tissues. This is a body defensive reflex to remove the injurious force. ${ }^{1}$ There are numerous elements of an allergic response that may lead to the resulting effects and tissue damage. Edema, infiltration of immune cells, and the accumulation of granulom as represent certain incendiary components. ${ }^{2}$ It's a protective mechanism while. The diverse occurrences and facilitators associated in the hypersensitivity reaction may cause other responses or exacerbate these. Immune cytokine such as tumer necrosis factor, IL-6 and IL- $1 \beta$ are increased in state of inflammation. ${ }^{3}$ Chlorella vulgaris Beijerinck is a single-celled fresh-water algae super-food and is thought to be one of the planet's earliest life-forms. Since the 1960's Chlorella has been popular in Japan as a multi-supplement taken to maintain health through optimal nutrition. ${ }^{4}$ It is the most researched of all the algae. Initially research was focused on improving our understanding of photosynthesis, but since the 1970s the health benefits of Chlorella have been documented in vast collection of studies. ${ }^{5}$ Chlorella users report to experience more energy, improved physical appearance and protection from disease and illness. Infectious diseases are the leading cause of death world-wide and antibiotic resistance has become a global concern. ${ }^{6}$ The clinical efficacy of many existing antibiotics is being threatened by the emergence of multidrug-resistant pathogens. Many infectious diseases have been known to be treated with herbal remedies throughout the history of mankind. Unmatched availability of chemical diversity among natural products either as pure compounds or standardized plant extracts, provide unlimited opportunities for new drugs to lead in pharmaceutical industries. ${ }^{7}$ With an urgent need to discover new antimicrobial compounds, there is also need for the developing novel mechanisms of action for new and re-emerging infectious diseases. In recent years, marine natural product search has yielded a considerable number of drug candidates. ${ }^{8}$ The chemical compounds responsible for the antibacterial activity in algae have been variously identified as bromophenols, carbonyls, halogenated aliphatic compounds, terpenes, isoprenylated and brominated hydroquinones, as well as phlobatannins. ${ }^{9}$ Throughout this study we conducted photochemical screening of paw inflammation caused by Chlorella vulgaris and carrageenan by measuring paw length, oxidative stress and cytokine level.

\section{MATERIALS AND METHODS}

\section{MATERIALS}

\section{Collection of Plant extract}

Chlorella vulgaris extract was purchased from the authenticated source and identified by using different phytochemical test.

\section{Animal}

Wistar rats were used for current study. Animal were placed in polyethylene cages with free access of feed and water at standard temperature $22^{\circ} \mathrm{C}$ and relative humidity $65 \%$. The animals were maintained according to guidelines given by Committee for the Purpose of Control and Supervision of the Experiments on Animals (CPCSEA). The experimental protocols were approved by the IAEC.

\section{Drug and Chemicals}

Carrageenan (Sigma Aldrich), ibuprofen (purchased from local pharmacy store), Cytokine kits was purchased from Elab science, Egg albumin, Indomethacin (purchased from local pharmacy vender). 


\section{METHODS}

\section{Pharmacognostic studies}

Physicochemical parameters: According to Indian Pharmacopoeia, the determination of various physicochemical parameters such as total ash, acid insoluble ash, water soluble ash and loss on drying was determined. ${ }^{10}$

Loss on Drying (moisture content determination: Around $1 \mathrm{gm}$ of the powdered leaf plant was weighed appropriately in a tarred petri dish which was previously dried under stated IP conditions. Through gentle side shaking, the powder was distributed as uniformly as possible. It was refrigerated in the desiccator and weighed again. The dish was dried in an oven at $100-105^{\circ} \mathrm{C}$ for $1 \mathrm{hr}$. The drying deficit was measured using wind-dried powder product. ${ }^{11}$

Preliminary Phytochemical analysis: Preliminary phytochemical screening of the extracts for saponins (Foam test), glycosides(Benedict's test), alkaloids (Dragendorff's test), tannins (Ferric chloride test) and flavonoids (Lead acetate test) using standard phytochemical screening methods. ${ }^{12}$

\section{Anti-inflammatory activity of Chlorella vulgaris (using carrageenan induced paw edema)}

The animals were randomly divided into six groups each containing six animals.

Group 1- Normal

Group 2- Carrageenan

Group 3- Standard (treated with $40 \mathrm{mg} / \mathrm{kg}$ ibuprofen)

Group 4 and 6- treated with 50, 100 and $200 \mathrm{mg} / \mathrm{kg}$ extract of Chlorella vulgaris respectively.

All the test groups were treated $1 \mathrm{hr}$ before the injecting carrageenan. Paw edema was induced in rats' hind paw by injecting $0.1 \mathrm{ml}$ of $1.5 \%$ carrageenan solution. Carrageenan solution was prepared in saline before $24 \mathrm{hr}$ and stored at $2-4^{\circ} \mathrm{C} . .^{13}$

Measurement of paw volume: Paw volume was measured by using plethysmometer at $0 \mathrm{hr}$ before injecting carrageenan, 1,2 , and $4 \mathrm{hr}^{13}$

Preparation of Homogenate: Paw tissue was collected by using biopsy punch and stored in PBS. Tissue homogenate was prepared by using homogenizer and finally centrifuged by using cooling centrifuge. Finally homogenate was stored at $-60^{\circ} \mathrm{C}$ up to further experiment ${ }^{14}$

Estimation of Oxidative stress and Cytokine: Antioxidant enzyme like SOD, GSH, and LPO was determined according to available kit by using microplate reader. Concentration was determined by using standard curve. ${ }^{15,16}$ Proinflammatory cytokines like IL-6, TNF-alpha were determined according to manufacturer's protocol. Concentration was determined by using standard curve. ${ }^{17,18}$

\section{Anti-inflammatory activity of Chlorella vulgaris (using cotton pellet induced granuloma)}

Experimental design: The mice were divided into five groups of six animals in each group. The mice were anaesthetized and sterile cotton pellets weighing $10 \pm 1 \mathrm{mg}$ will implanted subcutaneously into both sides of the groin region of each rat.

Group 1- Served as disease control

Group 2- Standard (animals received indomethacin at a dose of $10 \mathrm{mgkg}-1 \mathrm{~b} . \mathrm{w}$ for the same period)

Group 3 and 5- The extract of Chlorella vulgaris at the concentration of 50,100 and $200 \mathrm{mg} / \mathrm{kg}$ was administered orally for seven consecutive days from the day of cotton pellet implantation

On $8^{\text {th }}$ day the animals were anaesthetized and the pellets together with the granuloma tissues were carefully removed and made free from extra- neous tissues. The wet pellets were weighed and then dried in an oven at $60^{\circ} \mathrm{C}$ for $24 \mathrm{hr}$ to constant weight, after that the dried pellets were weighed again. Increment in the dry weight of the pellets was taken as a measure of granuloma formation. ${ }^{14} \%$ Protection was calculated by using following formula

$$
\begin{aligned}
& \text { (mean weight of granuloma in control - } \\
& \text { mean weight of granuloma in test group) } \\
& \text { mean weight of granuloma in control group }
\end{aligned}
$$

\section{Anti-inflammatory activity of Chlorella vulgaris (using} egg albumin induced inflammation)

Experimental design: Total of 36 rats were randomly divided into 6 groups each containing 6 animals.

Group 1- Normal treated with saline

Group 2- Egg albumin (0.1\%) of $1 \%$ solution prepared in saline

Group 3- Standard (ibuprofen $-40 \mathrm{mg} / \mathrm{kg}$ )

Group 4 and 6- Treated with 50, 100 and $200 \mathrm{mg} / \mathrm{kg}$ extract of Chlorella vulgaris

Inflammation was induced in rat by the sub plantar injection of egg albumin $(0.1 \mathrm{~mL})$ into right hind paw. The linear circumference of the injected paw was measured before and $0.5,1,2,4$ and $6 \mathrm{hr}$ after the administration of the egg albumin.

\section{RESULTS}

\section{Preliminary Phytochemical Analysis}

Preliminary phytochemical screening of methanolic extract of leaves shows the presence of high concentration of saponins, glycosides, alkaloids. While Tannins and flavonoids was absent in metanolic extract shown in Table 1.

\section{Effect of CV on carrageenan induced paw edema}

In this review, we observed that the carrageenan injected community showed an improvement in the volume of the paw and a single dose treatment with Xanthium Indicum based on Carrageenan-induced paw inflammation. This effect was however less effective than the standard drug shown in Figure 1.

Effect of CV on Oxidative stress: We used the paw homogenate to estimate the extent oxidative stress in carrageenan persuaded a state of obvious oxidative stress as apparent from a noteworthy increase in MDA level and a momentous decrease in the GSH and SOD paw tissue homogenates. The rat treated with test drug showed increase in the content of GSH and SOD. MDA level was found decreased in test drug treated group shown in Figure 2.

Effect of $\mathrm{CV}$ on proinflammatory cytokines: In this study we found that carrageenan treated group shows increase in the cytokine level as compared to the normal group. Standard drug treated rat's shows decrease in the cytokine level (TNF- $\alpha$, IL- 6 and IL-1 $\beta$ ). The entire treatment group shows decrease in the level of cytokine as compared to the

\begin{tabular}{ccc}
\multicolumn{2}{c}{ Table 1: Preliminary Phytochemical Analysis of Chlorella vulgaris. } \\
\hline Components & Test & Result \\
\hline Saponins & Foam test & Absent (-) \\
Glycosides & Benedict's test & Present $(+)$ \\
Alkaloids & Dragendorff's test & Present (+) \\
Tannins & Ferric chloride test & Absent $(-)$ \\
Flavonoids & Lead acetate test & Present $(+)$ \\
\hline
\end{tabular}




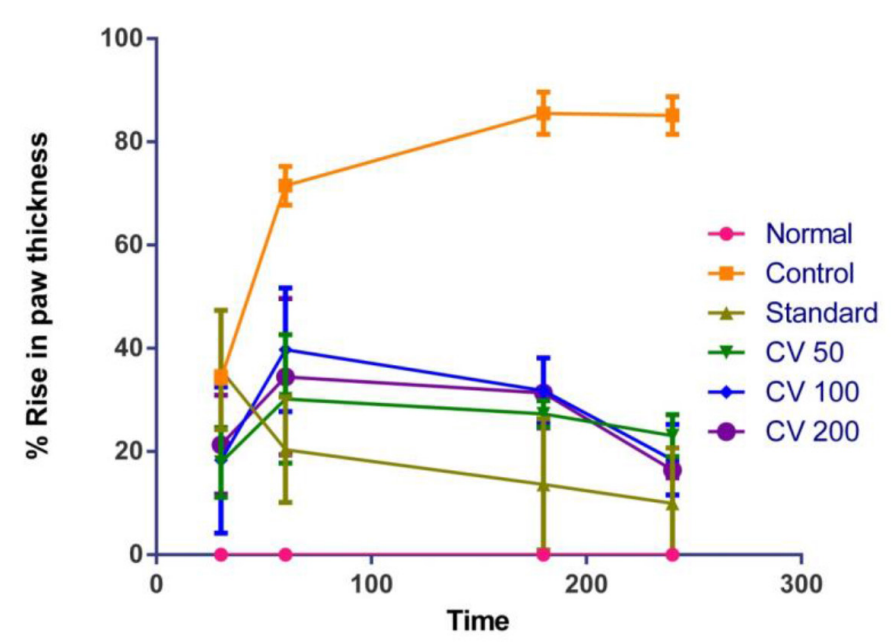

Figure 1: Effect of CV extract on \% rise in paw volume in carrageenan induced paw inflammation.

Data was analyzed by repeated measure ANOVA followed by Dunnet test and values are given in (Mean \pm SEM) $p$ valve 0.005 is considered as significant. (CV 50 - Chlorella vulgaris $50 \mathrm{mg} / \mathrm{kg}, \mathrm{CV}$ 100-Chlorella vulgaris $100 \mathrm{mg} / \mathrm{kg}, \mathrm{CV} 200$ Chlorella vulgaris $200 \mathrm{mg} / \mathrm{kg}$ ).

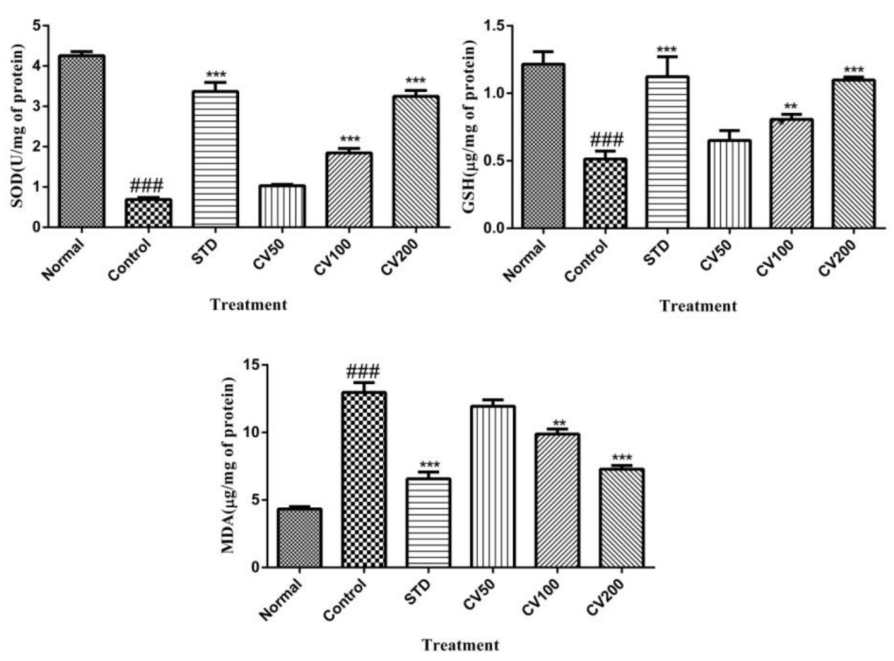

Figure 2: Effect of CV extract on Oxidative stress in carrageenan induced paw inflammation Data was analyzed by One Way ANOVA followed by Dunnet test and values are given in (Mean \pm SEM) $p$ valve 0.005 is considered as significant.

control group. Chlorella vulgaris treated with $200 \mathrm{mg} / \mathrm{kg}$ shows most prominent effect as compared to other group given in Figure 3.

\section{Effect of Chlorella vulgaris on Cotton pellet induced granuloma}

In this animal model of cotton pellet granuloma, inflammation and granuloma developed during a period of several days. The effects of CV on the proliferative phase of inflammation are shown in Table 2. When Chlorella vulgaris were tested for anti-inflammatory activity, CV markedly inhibited granuloma formation surrounding the pellet compared with vehicle control group by decreasing wet and dry weight of cotton pellet. Chlorella vulgaris administered at dose $200 \mathrm{mg} / \mathrm{kg}$ inhibition was

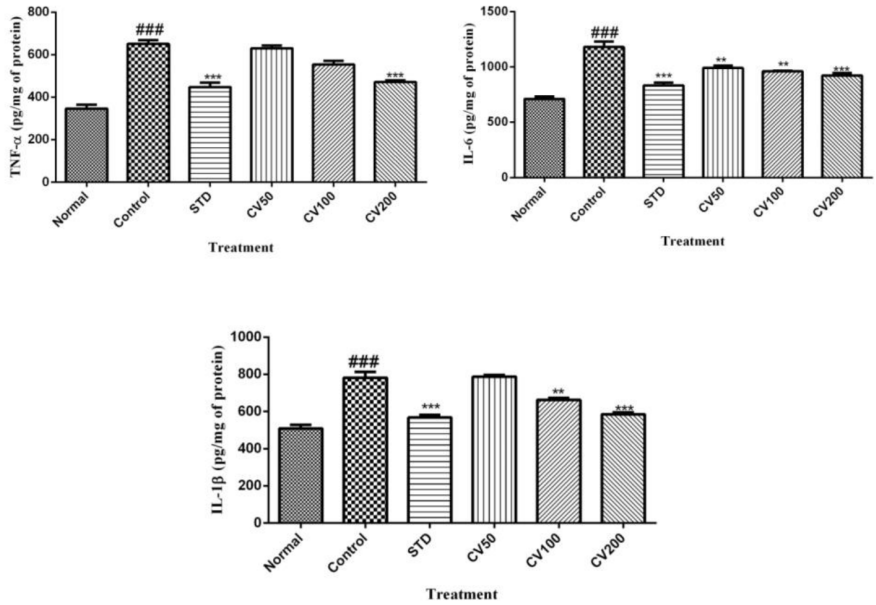

Figure 3: Effect of CV extract on proinflammatory cytokines in carrageenan induced paw inflammation.

Data was analyzed by One Way ANOVA followed by Dunnet test and values are given in (Mean \pm SEM) $p$ valve 0.005 is considered as significant. (CV 50- Chlorella vulgaris $50 \mathrm{mg} / \mathrm{kg}$, CV 100- Chlorella vulgaris $100 \mathrm{mg} / \mathrm{kg}$, CV 200-Chlorella vulgaris $200 \mathrm{mg} / \mathrm{kg}$ )

Table 2: Effect of CV on Wet and Dry Weight of Granuloma in Cotton Pellet Granuloma in Mice.

\begin{tabular}{ccccc}
\hline Group & $\begin{array}{c}\text { Wet } \\
\text { weight of } \\
\text { granuloma } \\
(\mathbf{m g})\end{array}$ & $\begin{array}{c}\text { Percentage } \\
\text { Inhibition }\end{array}$ & $\begin{array}{c}\text { Dry } \\
\text { weight of } \\
\text { granuloma } \\
(\mathrm{mg})\end{array}$ & $\begin{array}{c}\text { Percentage } \\
\text { Inhibition }\end{array}$ \\
\hline Control & $170 \pm 2.198$ & 00 & $54.25 \pm 1.652$ & 00 \\
Standard & $129.3 \pm 0.75$ & 23.94118 & $32.5 \pm 0.645$ & 40.09217 \\
CV 50 & $168 \pm 1.683$ & 1.176471 & $52.75 \pm 1.25$ & 2.764977 \\
CV 100 & $158.3 \pm 1.931$ & 6.882353 & $44.75 \pm 1.031$ & 17.51152 \\
CV 300 & 144.8 & 14.82353 & $38 \pm 0.9129$ & 29.95392 \\
\hline
\end{tabular}

Data was analyzed by One way ANOVA and represents as Mean \pm SEM, Dunnett's multiple comparision test, ${ }^{*} P<0.05,{ }^{*} P<0.01$ compared with control group (CV 50- Chlorella vulgaris $50 \mathrm{mg} / \mathrm{kg}$, CV 100- Chlorella vulgaris $100 \mathrm{mg} / \mathrm{kg}$, CV 200- Chlorella vulgaris $200 \mathrm{mg} / \mathrm{kg}$ ).

$14.82 \%$. Indomethacin inhibited the dry wet of granuloma at $40.09 \%$. Chlorella vulgaris treated group also showed dose dependent activity. The results obtained in this model were significant with $P<0.001$, for wet weight inhibition and $P<0.0002$, for dry weight inhibition of cotton pellet granuloma given in Table 2.

\section{Effect of Chlorella vulgaris on Egg albumin induced paw edema}

In this experiment, we observed that the egg albumin injected rat showed an increase in the volume of the paw and a single dose treatment with Chlorella vulgaris based on egg albumin-induced paw inflammation showed improvement in protection of paw volume increase. Chlorella vulgaris treated with $200 \mathrm{mg} / \mathrm{kg}$ showed good effect as compared to other test group. This effect was however less effective than the standard drug shown in Figure 4. 


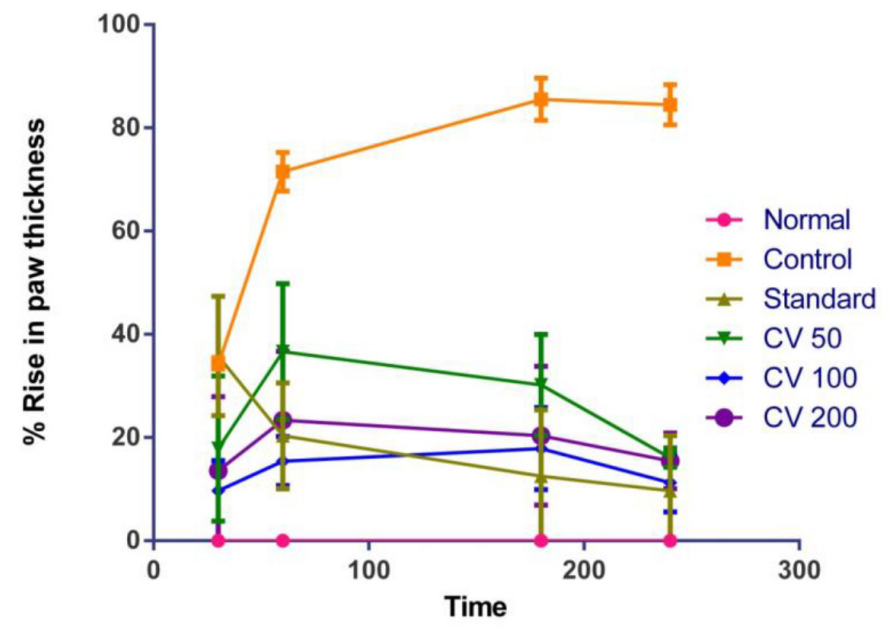

Figure 4: Effect of $C V$ extract on $\%$ rise in paw volume in egg albumin induced paw inflammation.

Data was analyzed by repeated measure ANOVA followed by Dunnet test and values are given in (Mean \pm SEM) $p$ valve 0.005 is considered as significant. (CV 50- Chlorella vulgaris $50 \mathrm{mg} / \mathrm{kg}$, CV 100- Chlorella vulgaris $100 \mathrm{mg} / \mathrm{kg}$, CV 200-Chlorella vulgaris $200 \mathrm{mg} / \mathrm{kg}$ )

\section{DISCUSSION}

Present study based on phytochemical and pharmacological investigation of Chlorella vulgaris. In attempt to have a scientific finding about the use of Chlorella vulgaris, we examined the anti-inflammatory behavior of the extract in carrageenan induced paw edema in rats. The analyze data clearly showed that extracts have anti-inflammatory activity due to the hugely significant reactions of certain extracts to inhibit the emergence of oedema following carrageenan subplantar injection. ${ }^{13,19}$ The extracts, that indicated the greatest anti-inflammatory activity, often showed highly significant statistical values. In preliminary phytochemical screening it was found that plant extract contains saponins, glycosides, alkaloids, while tannins and flavonoids are absent in extract. Less value of moisture contents means there is no growth of bacterial, fungal and yeast. In carrageenan injected paw showed that constant swelling started from 30 min same reported in many published research articles. ${ }^{14}$ In case of oxidative stress it was found that increase in the level of MDA and decrease in SOD and GSH in paw homogenate in control group it is evidence of inflammation and same plant extract treated group showed decrease in MDA level and increase in SOD and GSH level. From this result we proved that plant has strong anti-oxidant activity. In cytokine estimation it was found that elevated level of cytokine like TNF-alpha, IL-6 and IL-1beta in control group while extract treated group showed decrease in level of all cytokine. Elevation of cytokine mainly found in the inflammation and pain. ${ }^{20}$ In case of egg albumin induced inflammation, rat showed increased paw thickness and rats treated with Chlorella vulgaris normalized the paw thickness. In chronic inflammation was induced in mice by implanting cotton pellet granuloma in mice. Control group showed increased dry and wet weight of granuloma, while mice treated with Chlorella vulgaris showed decreased dry and wet weight of granuloma.

\section{CONCLUSION}

The study's findings have shown that different phytochemicals are available in plant extracts and could be accountable for pharmacological activity. Extract reduces inflammation in rats, resulting in the conclusion that plant extract has potent anti-inflammatory activity.

\section{CONFLICT OF INTEREST}

The authors declare that there is no conflict of interest.

\section{REFERENCES}

1. Medzhitov R. Origin and physiological roles of inflammation. Nature. 2008;454(7203):428-35. doi: 10.1038/nature07201, PMID 18650913.

2. Bennett JM, et al. Inflammation-nature's way to efficiently respond to all types of challenges: implications for understanding and managing 'the epidemic' of chronic diseases. Fron. Med. 2018;5:316.

3. Vane J, Botting R. Inflammation and the mechanism of action of anti-inflammatory drugs. FASEB J. 1987;1(2):89-96. doi: 10.1096/fasebj.1.2.3111928, PMID 3111928.

4. Lin CC, Shieh DE. The anti-inflammatory activity of Scutellariari vularis extracts and its active components, baicalin, baicalein and wogonin. Am J Chin Med. 1996;24(1):31-6. doi: 10.1142/S0192415X96000050.

5. Hossan ANMF, Zaman F, Barman MR, et al. Antinociceptive activity of Xanthium indicum J. Koenig ex Roxb. (Asteraceae) leaves and Leucas aspera (Willd.) Link (Lamiaceae) whole plants. Adv Nat Appl Sci. 2011;5(2):214-7.

6. Liu ZY, Wang GC, Zhou BC. Effect of iron on growth and lipid accumulation in Chlorella vulgaris. Bioresour Technol. 2008;99(11):4717-22. doi: 10.1016/j. biortech.2007.09.073, PMID 17993270.

7. Lim SL, Chu WL, Phang SM. Use of Chlorella vulgaris for bioremediation of textile wastewater. Bioresour Technol. 2010;101(19):7314-22. doi: 10.1016/j. biortech.2010.04.092, PMID 20547057.

8. Widjaja A, Chien CC, Ju YH. Study of increasing lipid production from fresh water microalgae Chlorella vulgaris. Journal of the Taiwan Institute of Chemica Engineers. 2009;40(1):13-20. doi: 10.1016/j.jtice.2008.07.007.

9. Kula M, Rys M, Mozdzen K, Skoczowski A. Metabolic activity, the chemical composition of biomass and photosynthetic activity of Chlorella vulgaris under different light spectra in photobioreactors. Eng Life Sci. 2014;14(1):57-67. doi: 10.1002/elsc. 201200184

10. Sharma A, et al. Preliminary phytochemical screening of fruit peel extracts of Annona squamosa Linn. J Curr Pharma. Resources. 2013;4(1):1038.

11. Aiyegoro OA, Okoh Al. Preliminary phytochemical screening and in vitro antioxidant activities of the aqueous extract of Helichrysumlongifolium DC. BMC Complement Altern Med. 2010;10(1):21. doi: 10.1186/1472-6882-10-21, PMID 20470421.

12. Basma AA, Zakaria Z, Latha LY, Sasidharan S. Antioxidant activity and phytochemical screening of the methanol extracts of Euphorbia hirta L. Asi Paci J Tro. Med. 2011;4(5):386-90.

13. Ratheesh M, Helen A. Anti-inflammatory activity of Ruta graveolens Linn on carrageenan induced paw edema in Wistar male rats. Afr J Biotechnol. 1990;6(10):125-9.

14. Chi SC, Jun HW. Anti-inflammatory activity of ketoprofen gel on carrageenaninduced paw edema in rats. J Pharm Sci. 1990;79(11):974-7. doi: 10.1002/ jps.2600791106, PMID 2292773.

15. Shinde SD, et al. The Berberis aristata Ameliorates oxazolone induced contact dermatitis: in-vivo and in silico evidences. Adv Trad Med 2020: 1-8.

16. Jain PG, Mahajan UB, Shinde SD, Surana SJ. Cardioprotective role of FA against isoproterenol induced cardiac toxicity. Mol Biol Rep. 2018;45(5):1357-65. doi: 10.1007/s11033-018-4297-2, PMID 30105550.

17. Sonawane VK, Mahajan UB, Shinde SD, Chatterjee S, Chaudhari SS, Bhangale HA, Ojha S, Goyal SN, Kundu CN, Patil CR. A chemosensitizer drug: disulfiram prevents doxorubicin-induced cardiac dysfunction and oxidative stress in rats. Cardiovasc Toxicol. 2018;18(5):459-70. doi: 10.1007/s12012-018-9458-y, PMID 29779119

18. Masule MV, et al. Evaluation of antioxidant and anti-parkinsonism activity of betaine in experimental rats. J Drug Deli Ther. 2019;9(2-s):417-21.

19. Tsai CC, Lin CC. Anti-inflammatory effects of Taiwan folk medicine 'Teng-Khia-U' on carrageenan- and adjuvant-induced paw edema in rats. J Ethnopharmacol. 1998;64(1):85-9. doi: 10.1016/S0378-8741(98)00108-1.

20. Hussein SZ, Mohd Yusoff K, Makpol S, Mohd Yusof YA. Gelam honey inhibits the production of proinflammatory, mediators NO, PGE2, TNF- $\alpha$, and IL-6 in carrageenan-induced acute paw edema in rats. Evi Bas Complem Alter. Med. 2012;2012:1-14

Article History: Submission Date : 22-08-2021; Revised Date : 13-09-2021; Acceptance Date : 30-09-2021.

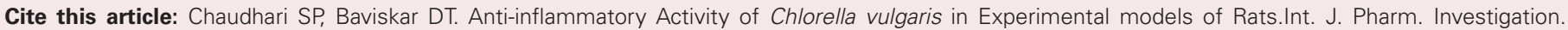
$2021 ; 11(4): 358-61$ 